\title{
An Image of a Highly Relativistic Jet from a Large Flare in the X-Ray Binary Cyg X-3
}

\section{A. J. Mioduszewski}

JIVE/National Radio Astronomy Observatory, Socorro, NM 87801, U.S.A.

R. M. Hjellming \& M. P. Rupen

National Radio Astronomy Observatory, Socorro, NM 87801, U.S.A.

E. B. Waltman

Naval Research Laboratory, Washington, DC 20375, U.S.A.

G. G. Pooley

Mullard Radio Astronomy Observatory, Cambridge, CBЗ OHE, U.K.

F. D. Ghigo

National Radio Astronomy Observatory, Green Bank, WV 24944, U.S.A.

R. P. Fender

Astronomy Centre, University of Sussex, Palmer, Brighton, BN1 9QH, U.K.

Abstract. A VLBA image of the X-ray binary Cyg X-3 made two days after a 10 Jy radio flare shows a one-sided, curved jet with a size scale of $\approx 60$ mas. This seems to indicate a jet with a velocity of $\geq 0.89 \mathrm{c}$ with a inclination of a few degrees to the line of site, and indicates that some of events where Cyg X-3 flares above $3 \mathrm{Jy}$ may come from highly relativistic jets close to the line of sight.

\section{Introduction}

Cyg X-3 is an X-ray binary with an X-ray and IR period of 4.8 hours. Since it was first seen to have a 22 Jy flare in 1972 (Hjellming \& Han 1995), it has had radio flares above $3 \mathrm{Jy}$ at an average rate of 1.1 per year. Its normal "quiescent" radio state is continually variable at levels of $0.1-0.3 \mathrm{Jy}$. However, before the very large radio flares it changes to a "quenched" radio state at a level of about $10 \mathrm{mJy}$. As shown by McCullough et al. (1997), pre-flare quenching in hard $\mathrm{X}$-rays (20-100 keV) also occurs, and often precedes, a large radio flare. After some large flares the radio source has been resolved with a N-S rate of expansion that has been estimated at about 5 mas/day. VLBA imaging by Schalinski et al. (1996) after a flare showed two, and sometimes three, components.

\section{January - February 1997 Observations of Cyg X-3}

On Feb. 6, 8, and 11 the first VLBA observations of Cyg X-3 after a $10 \mathrm{Jy}$ flare that peaked on Feb. 4, were made at $15 \mathrm{GHz}$. As with many Cyg X-3 flares, about 40 days in a quenched HXR state and about three days in a radio quenched state were followed by a weak precursor flare to $0.5 \mathrm{Jy}$, a stronger precursor flare to $5 \mathrm{Jy}$, and then a larger flare to $10 \mathrm{Jy}$. The image made from Feb. 6 data, two days after the peak at $10 \mathrm{Jy}$, is shown in Figure 1. 


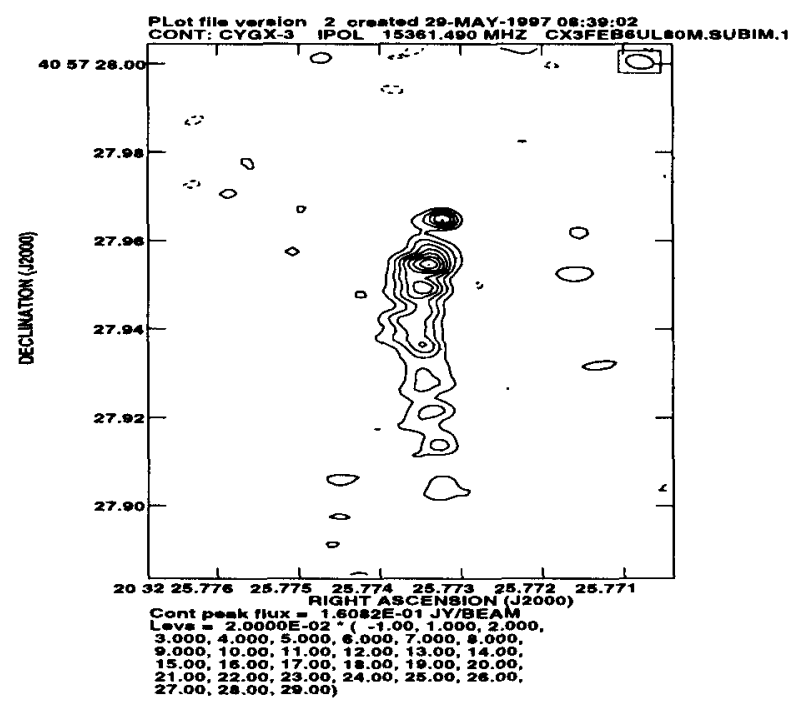

Figure 1. A VLBA image of the X-ray binary Cyg X-3 made from Feb. 6, 1997 data at $15 \mathrm{GHz}$, two days after a $10 \mathrm{Jy}$ flare.

\section{Analysis and Conclusions}

The image in Figure 1 appears like the "classical" VLB jets seen in distant quasars, where the observed side is a Doppler-boosted, approaching jet close to the line of sight, and the receding jet is too weak to be seen. Identifying the upper, most compact source as the "core," one can estimate the properties of the twin-jet by assuming the approaching proper motion is $\approx 5 \mathrm{mas} / \mathrm{day}$, the ratio of approaching to receding flux is $\geq 750$ from the image in Figure 1, and the distance is $10 \mathrm{kpc}$; then the jet velocity is $\geq 0.89 \mathrm{c}$ and the inclination of the jet to the line of sight is $\leq 2^{\circ}$. These results indicate that at least some of the strong flare events in Cyg X-3 are like those that produce the $v=0.92 \mathrm{c}$ jets in the black hole X-ray transients GRS 1915+105 (Mirabel \& Rodríguez 1994) and GRO J1655-40 (Hjellming \& Rupen 1995). The fact that Cyg X-3 is the X-ray binary with the strongest radio flaring may be related to Doppler boosting of jets oriented close to the line of sight.

Acknowledgments. Mioduszewski acknowledges support for her research by the European Union under contract CHGECT920011. The National Radio Astronomy Observatory is a facility of the NSF, operated under a cooperative agreement by Associated Universities, Inc.

\section{References}

Hjellming, R. M. \& Han, M. 1995. in "X-Ray Binaries" (Cambridge University Press: Cambridge), 308-330.

Hjellming, R. M. \& Rupen, M. P. 1995. Nature, 375, 464-468.

McCullough, M. L. et al. 1997. in The Transparent Universe, ESA, SP-382, 265.

Mirabel, I. F. \& Rodríguez, L. F. 1994. Nature, 371, 46-48.

Schalinski, C. J. et al. 1996. ApJ, 447, 752-759. 